\title{
Research on Laboratory Construction and Management under the Environment of Dazhiyiyun
}

\author{
Shirong Qin ${ }^{a}$, Xiaojun Lu, ${ }^{b, *}$ \\ Harbin university of Commerce, Harbin, China \\ asdqsr@163.com, ${ }^{b}$ lxjharbin@126.com
}

Keywords: Informatization; Laboratory management; Networked laboratory

\begin{abstract}
Dazhiyiyun"is the abbreviation of Big Data, Intelligent, Mobile Internet and Cloud Computing. The laboratory is an important part of colleges and universities. With the development of science and technology and the promotion of connotative construction in colleges and universities, how to further strengthen the construction of sustainable development laboratories in the context of "Dazhiyiyun", so that laboratory teaching and construction follow the pace of the times, according to economic situation and industry.
\end{abstract}

\section{Introduction}

With the rapid development of information technology, education has entered a new era of informationization. Judging from the construction and management of domestic university laboratories, there is a general lack of openness, low level, and limited scope. In addition, students' enthusiasm for entering the laboratory is not high, and most students do not know how to use laboratory development. Personalized learning. Obviously, this is not conducive to improving the efficiency of laboratory use and equipment utilization, but also seriously affecting the quality of personnel training. How to strengthen the development and application of high-quality digital education resources and implement classroom construction has become an important research topic in the education of all stages of education in China and the development of various disciplines. As the most important practice space for college students, the laboratory is very important to the career development of college students. Under the background of "Dazhiyiyun", the construction of university laboratories urgently needs to introduce modern information technology such as cloud computing, big data, and Internet of Things to adapt to social development and modern college education reform. The laboratory construction and management of many applied undergraduate colleges still adopt the traditional mode, which relies on manual decentralized management, and the informationization process is relatively backward. There are some outstanding problems.

\section{Laboratory layout structure and resource allocation is not reasonable}

The strengthening of students' practical ability and the improvement of innovation and entrepreneurial quality put forward higher requirements for the experimental course content of the experimental teaching. On the one hand, due to historical reasons, there are many institutions in China's colleges and universities. The integration of experimental teaching resources is not enough, and the structural contradiction between laboratory space and instrument and equipment resources is prominent, which has affected the further expansion and construction of the laboratory. On the other hand, due to the limited investment, colleges and universities generally have the conditions for the teaching of public basic experiments in addition to the experimental teaching conditions of the major disciplines, especially the experimental teaching conditions of the newly-run majors and the actual needs of the cultivation of innovative talents. Compared with foreign excellent universities, the gap is still larger. The data of the average teaching and research equipment value and the average construction area of the experimental building need to be improved. Experimental sites, instruments 
and equipment, and inadequate experimental facilities are still a bottleneck restricting the creation of high-level universities with distinctive characteristics.

\section{Incompatibility of management system and operational mechanism}

In the management system, whether it is a secondary or tertiary management model, there is a phenomenon that the division of labor between schools and departments is unclear and the relationship is not straightened out. There are too many areas and links that the school or functional department should not manage, and the place where the management is not in place. In addition to different departmental functions, many colleges and universities have classified different types of laboratories into different functional departments according to the type of laboratories, and lacked a strong operational organization to coordinate planning laboratory construction and deployment of laboratory resources. The quality of laboratory resources cannot be effectively pooled. Leading to a narrow professional scale and a single function, it is not conducive to interdisciplinary and interdisciplinary experiments, and is not conducive to collaborative innovation of interdisciplinary subjects. Even some professional laboratories are seriously idle. Most of the research-based laboratories are divided into resources by a research group to form a single-player experimental team. This is in line with the need to build an interdisciplinary and interdisciplinary comprehensive platform for sustainable development of laboratory construction. Far away, we do not attach importance to the reform of the experimental teaching system, and even lack the management supervision of the corresponding functional departments to ensure the sharing of synergies, benign interaction and sustainable development of the laboratory's high-quality resources.

\section{Open shared network platform lags low existing resource sharing}

On the one hand, the traditional laboratory management system of colleges and universities is mainly based on manual methods, with a large workload, and there are drawbacks such as inquiries and low management efficiency. The low utilization rate of laboratories and instruments and equipment has caused waste of resources to some extent. The open and shared network platform is the product of technological development and the need of the market economy. A backward shared system will reduce resource utilization and cause repeated purchases. On the other hand, due to the lack of performance appraisal of laboratories and large-scale instruments and equipment, the open sharing of many laboratories and large-scale instruments and equipment is not high, and the laboratories are in a state of mutual closure, which makes it impossible to share existing resources. This makes the equipment and instruments unable to fully exert their practical value. Even some equipments are too protected due to management and are idle, which cannot fully play a role in the experiment.

\section{University laboratories have evolved from traditional single laboratories to comprehensive, multi-functional R\&D laboratories.}

In the era of “Dazhiyiyun”, big data analysis, artificial intelligence, mobile data and cloud services are becoming more and more important in current accounting graduate education. Traditional resource-oriented innovation and entrepreneurship education is gradually being replaced by opportunity-oriented innovation and entrepreneurship education. Therefore, in the information age, accounting graduate innovation and entrepreneurship education needs to collaboratively integrate various opportunity resources and change the traditional innovation and entrepreneurship education model.

\section{Strengthen top-level design to strengthen the construction of university laboratory resources}

To fully utilize the multi-faceted and multi-faceted integration and optimization of laboratory resources, it is necessary to strengthen the top-level design, and further rationalize the management 
system, clarify the division of functions, and strengthen responsibilities. It may be considered to establish a management system with the school management laboratory as the mainstay and the hospital management laboratory as the main body. Enhance the faculty's autonomy in the planning and management of laboratory construction, as well as the power to control the funds. The college level focuses on the unified planning, unified construction, and unified management mode based on the director of the experimental center and the professor of laboratory responsibility. Realize the unity of responsibility and responsibility for laboratory construction and management. At the school level, we should focus on macro-level management and regulatory evaluation, and provide quality services to the department.

\section{Ensure that university laboratories should be scalable}

In order to ensure the construction of a university laboratory with a high level of comprehensiveness, it must have good scalability in hardware and software equipment. On the one hand, with the advancement of teaching, the upgrading of software and hardware, the university laboratory must always maintain high efficiency and advancement. The various equipments and application software of the laboratory must be compatible, and it must meet the current experiment. Need, should also have the ability to update and upgrade with the development of technology in the future to ensure laboratory teaching and experimental exploration. On the other hand, to build high-level, comprehensive university laboratories, the equipment selected should have strong openness, ensuring that network interconnection and network compatibility are well realized.

\section{Laboratory management of information technology construction refinement and flexible management}

The informatization construction of laboratory management mainly includes the informationization of experimental teaching management, the informationization of laboratory equipment management, and the informationization of laboratory teaching process management. Through these measures, the traditional manual management mode is reformed, making laboratory management work and experimental teaching work more convenient, more efficient and more standardized.

Do a comprehensive statistical work on the materialized laboratory resources, and establish a dynamic networked database; the materialized resources with different characteristics should be intensively managed, pay attention to detail awareness, service awareness and system awareness, and take targeted measures. At the same time, it is necessary to make full use of the "Dazhiyiyun" technology to build a comprehensive experimental teaching platform with diversified forms, and gradually realize the transformation of the experimental teaching platform from the teaching-affiliated laboratory to the teaching and research and social service platform, and achieve diversified development direction. In the laboratory team and laboratory culture construction, it should be based on the flexible management of people-oriented.

\section{Building an exchange platform based on the informationization of teaching resources}

In the process of laboratory information construction, relevant teaching resources should also be fully informatized, and the experimental and experimental contents should be processed through video, animation, voice, pictures, text, and PPT courseware. The communication network or multimedia device can visually display the teaching content to the students, which can effectively improve the teaching efficiency of the teacher, and can also improve the students' interest and learning effect through such an intuitive, convenient and lively form. The smooth flow of information is the condition for the sharing of laboratory resources. To realize the sharing of laboratory resources, it is necessary to introduce an intelligent management system and build a platform for laboratory resource information exchange. Today, with a high degree of informatization, Internet technology 
has greatly facilitated the exchange of laboratory resources information. The open network information exchange platform of the laboratory not only facilitates the sharing of laboratory space and laboratory equipment between teachers and students of different colleges, but also makes it possible to realize laboratory resource sharing among universities, schools and enterprises.

\section{Networked laboratory and virtual laboratory combination}

The virtual simulation lab has the following outstanding advantages: 1 Saving money and space. 2 The teaching style is flexible and diverse, and the teaching effect is improved. 3 Construction and maintenance costs are low and scalability is good. Therefore, a variety of professional virtual simulation software commonly used in the profession is introduced into the laboratory to establish a virtual experimental platform. In the experimental training, according to the theory-simulation-physical steps, the students are guided to complete a complete development design process. The practical ability to do things is very beneficial.

On the basis of this, in order to facilitate centralized management and improve efficiency, it can be informatized and networked, and the various links of experimental teaching can be connected through the network to build a communication platform and connect the separate laboratories. A whole, providing a hardware platform for the information management of the laboratory.

\section{Acknowledgements}

This project is supported by the practical teaching reform and research project of Harbin Commercial University in 2018 (subject name: mobile laboratory design and construction and application) and the higher education teaching reform research project of Heilongjiang Education Department in 2018 (subject name: mobile laboratory design and construction and application).

\section{References}

[1] Liu Xinxin, Wang Chengyong, Wu Fugen, Chen Langcheng, Thoughts and Suggestions on the Construction and Management of University Laboratories under the Background of "Collaborative Innovation"-Inspiration from the Investigation of Two Universities in Germany and France, Experimental technology and management, Vol. 31 No. 8 Aug. 2014.

[2] Qun Bao, Min Shao, Dali Yang. Environmental Regulation, Provincial Legislation and Pollution Emission in China. Economic Research Journal. Vol.48 (2013) p.42-54.

[3] Shuo Chen, Ting Chen. Air Pollution and Public Health:Evidence from Sulfur Dioxide Emission of Coal-fired Power Stations in China. Economic Research Journal. Vol.49 (2014) p.158-169.

[4] Zhengge Tu, Renjun Kan. Industrialization's, Urbanization's Dynamic Marginal Carbon Emissions The Analytical Framework Based on LMDI "Two-Level Perfect Decomposition" Method. China Industrial Economics. Vol.30 (2013) p.31-43.

[5] Yang Ping, Yang Chaofeng, Liu Hongbo, Intelligent Construction and Management of University Laboratories, Vol.20 No. 6 Dec.2017.

[6] Zhang Liucheng. Research of Innovation and Entrepreneurship Education in Harbin University of Commerce [J]. Social Science and Humanity, 2015(9), pp. 22-24.

[7] Zhang Liucheng. Application and Analysis of Witkey Mode in Network Economy [J]. Advances in Social Science, Education and Humanities Research, 2015(9), pp. 102-105.

[8] Lin Wei, Discussion on the Construction Path of University Innovation and Entrepreneur, Vol. 34 No. 2 Feb. 2017.

[9] Zhang Liucheng. The Research and Innovation of Witkey Mode under the New Era [J]. Advances in Education Research, 2015(11), pp. 102-105. 
[10] Zhang Liucheng. Research on the problems of the application of XBRL financial statement in small and medium sized enterprises [J]. Advances in Social Science, Education and Humanities Research, 2016(4), pp. 1421-1424.

[11] Zhang Liucheng.Application-oriented Institutes Innovation Thinking and Practice of Talent Training Mode [J]. Advances in Social Science, Education and Humanities Research, 2016(4), pp. 1425-1429.

[12] Zhang Hongyu, Exploration and Practice of Laboratory Management in Local Universities, Vol. 33 No.7 Jul. 2016. 